\title{
Providing Local Information for Search and Rescue Using Sensor-Based Local Databases
}

E. Ergen ${ }^{1}$, S. Sariel-Talay ${ }^{2}$, G. Guven ${ }^{3}$

${ }^{1}$ Civil Engineering Department, Istanbul Technical University, Istanbul, Turkey, 34469; PH \& FAX (+90) 212 285-6912; email: esin.ergen@itu.edu.tr

${ }^{2}$ Computer Engineering Department, Istanbul Technical University, Maslak, Istanbul, Turkey, 34469; PH (+90) 212 285-3852; FAX (+90) 212 285-3679; email: sariel@itu.edu.tr

${ }^{3}$ Civil Engineering Department, Istanbul Technical University, Istanbul, Turkey, 34469; PH \& FAX (+90) 212 285-6912; email: guvengurs@itu.edu.tr

\begin{abstract}
This paper presents the design of a system that is being developed to utilize wireless local databases for making necessary local information readily available to search and rescue teams following a natural disaster. The Search and Rescue Data Access Point (SR-DAP) system is designed for local data storage and retrieval using sensor nodes deployed on the exterior sides of buildings. The paper reports on the results of the interviews conducted with experts to identify types of information items that are needed during search and rescue operations. Also, memory requirements for identified information groups are given. The implementation details of the developed system are provided and initial findings of data reading/writing tests performed in a laboratory environment are presented. The technical feasibility of using wireless sensors for local data storage and retrieval is discussed in the light of the initial findings.
\end{abstract}

Keywords: disaster, sensors, local data storage

\section{Introduction}

Availability of information related to buildings and their residents is an important factor that affects not only the time spent for the search and rescue (S\&R) efforts but also the effectiveness of the S\&R operations. Currently some information about buildings, their contents and residents are stored in centralized databases. However, S\&R teams cannot rely on accessing this information from those databases since information infrastructure is usually damaged, unreliable or overloaded immediately 
after a disaster, such as an earthquake (Worldbank 1999, Ozerdem and Jacoby 2005, Ito 2006). Consequently, S\&R teams collect the related local information intuitively through site observations and by talking to people in the neighborhood who are familiar with the buildings. This process is time-consuming; also the information is unreliable and incomplete due to the fact that local people can still be under trauma of the disaster. More information on how current S\&R operations are performed following a disaster (i.e., an earthquake) can be found at Ergen et al. (2008).

In the ongoing research study explained in this paper, utilization of wireless sensor based local databases were proposed for making necessary local information readily available and easily accessible to S\&R team members. Sensor nodes, which consist of sensing, data processing and communicating components, can monitor physical or environmental conditions at different locations and are capable of constituting a wireless sensor network (Akyildiz et al. 2002). The challenge in using wireless sensors in the study explained in this paper lies in transmission and storage of large size of media information which increases with respect to number of residents. This paper gives an overview of the developed system, called Search and Rescue Data Access Point, and identifies the information to be stored in the nodes. It also discusses the technical feasibility of using wireless sensors as a local data storage unit based on these challenges. More detailed descriptions of current S\&R practice and its limitations, and data transmission process in the system can be found at Ergen et al. (2008) and Sariel et al. (2008).

\section{Background}

Existing disaster management systems are mostly developed for identification, prediction and recovery phases and do not support real-time response activities (Pradhan et al. 2007). In the recent studies, researchers proposed several disaster management system frameworks (Asama et al. 2006, Chen et al. 2007, Pradhan et al. 2007); some of which focus on establishing an effective ad-hoc communication among mobile devices to be used during S\&R by the responders (Asama et al. 2006, Chen et al. 2007). Geographic information system (GIS), web collaboration tools, robots, sensors, building blackbox system and radio frequency identification technology (RFID) are various components that were incorporated within these frameworks. The proposed SR-DAP system do not incorporate any advanced building sensing and control systems or web collaboration tools considering that these systems might not be properly working during a disaster. Instead a stand alone sensor based local data storage system is designed which is easily deployable in current buildings and does not rely on other systems.

Wireless sensors are usually used in networks for a variety of applications such as environmental monitoring, infrastructure management, and public safety. Each sensor has some data processing capability, a radio frequency transceiver, a power source and memory. A typical sensor network has multiple nodes and is capable of routing data to a sink node. When compared with other local data storage technologies such as barcode and radio frequency identification, the advantages of using a sensor for storing local information are larger memory (i.e., $1 \mathrm{MB}$ ) and data routing capabilities which enables access to data that is stored at other nodes. 


\section{Overview of the Identified Information Items}

The proposed SR-DAP system utilizes wireless sensor nodes for storing necessary information items locally and making them readily available to the S\&R teams following a disaster. More detailed information about the proposed system can be found at Ergen et al. (2008).

To identify necessary local information items needed for S\&R operations after a disaster, S\&R guidelines were used (FEMA 2003, INSARAG, 2006) and interviews were conducted with thirteen experts from Istanbul Technical University, Turkish Civil Defense Association and Turkey Search and Rescue Association (AKUT), which are leading organizations in their areas of specialization. Local information items that were identified are related with the neighborhood, buildings and their residents. These information items are classified into two main groups: (1) information stored before a disaster, (2) information stored after a disaster (assessment information). The former includes information about the neighborhood, buildings and residents and will be used to search buildings and rescue people efficiently and effectively. The latter consists of information related to the rescued or trapped residents and the condition of the building. This information will be used by recovery teams or by the other S\&R teams who checks the same building.

The information items which needs to be stored before a disaster will be recorded at two different locations at two levels of detail: (1) neighborhood information includes high level information about the neighborhood, buildings in the neighborhood and their residents, and this information will be stored at local public building, (2) building information includes detailed information related to the building where this information was stored at and related to the residents of this building. Neighborhood information gives an overview of the area and helps the S\&R teams in making effective decisions about which buildings have priorities. Neighborhood information consists of the following information items and groups: transportation map of the neighborhood, layout plan of the neighborhood, building types and total number of people living in each building.

Building information provides required information about a building and its residents, and assists the S\&R team in developing a strategic operation plan (e.g., for selecting the right equipment). The building information includes type of building, hazardous materials, the layout plans of the building, utility shut-off locations, the number of floors, the type of the structural system (e.g., concrete), the age of the building, total number of people staying in the building within specific time periods (e.g., during daytime and nighttime). Building information also includes information about the residents which consists of residents' personal information (each person's name, age, health information etc.), number of residents living in each apartment in the building and the time period when they stay in the building.

Assessment information, which is the second group of information to be stored locally, is created by $S \& R$ teams after completing the $S \& R$ operations. Assessment information includes the name of the search team, time and date of the team's entrance and exit to/from the building, whether other teams can enter the building (go/no go information), residents' names, conditions (e.g., live or dead) and their locations, and hazardous situations in a building. Assessment information is stored to keep an accurate record of the performed activities to inform recovery teams who 
perform the debris removal or other S\&R teams who might later check the building for potential survivors.

\section{SR-DAP System Architecture}

SR-DAP system is designed for storage and retrieval of local information on/from sensor nodes deployed on exterior walls of buildings to assist S\&R operations. The chance of getting a heavy damage on a local sensor placed on a building is low since those sensors are protected in durable encasings. Data redundancy can also be created by storing the same data in multiple sensors so that if a sensor is damaged, the data would be available in other sensors.

Figure 1 illustrates the main components of the SR-DAP system and how they are used in different phases. At each neighborhood, wireless sensors are placed at the exterior side of (1) a local public building to store neighborhood information (Figure 1a) (2) each building to store building information (Figure 1b). Information that is necessary for S\&R operations needs to be entered to the sensors beforehand. To retrieve/store data from/on the sensor nodes, a handheld computer (i.e., a PDA or laptop) integrated with a sink sensor node will be carried by the S\&R teams. Following a disaster, S\&R teams query the sensor nodes to retrieve the related neighborhood or building information (Figure 1c and 1d) and finally enter assessment information to a sensor attached to a building once S\&R is completed at that building (Figure 1d). A local database is assumed to exist in the computing hardware of operators/rescuers for encoding data that is stored in the nodes. More information on how the system is used can be found at Ergen et al. (2008).

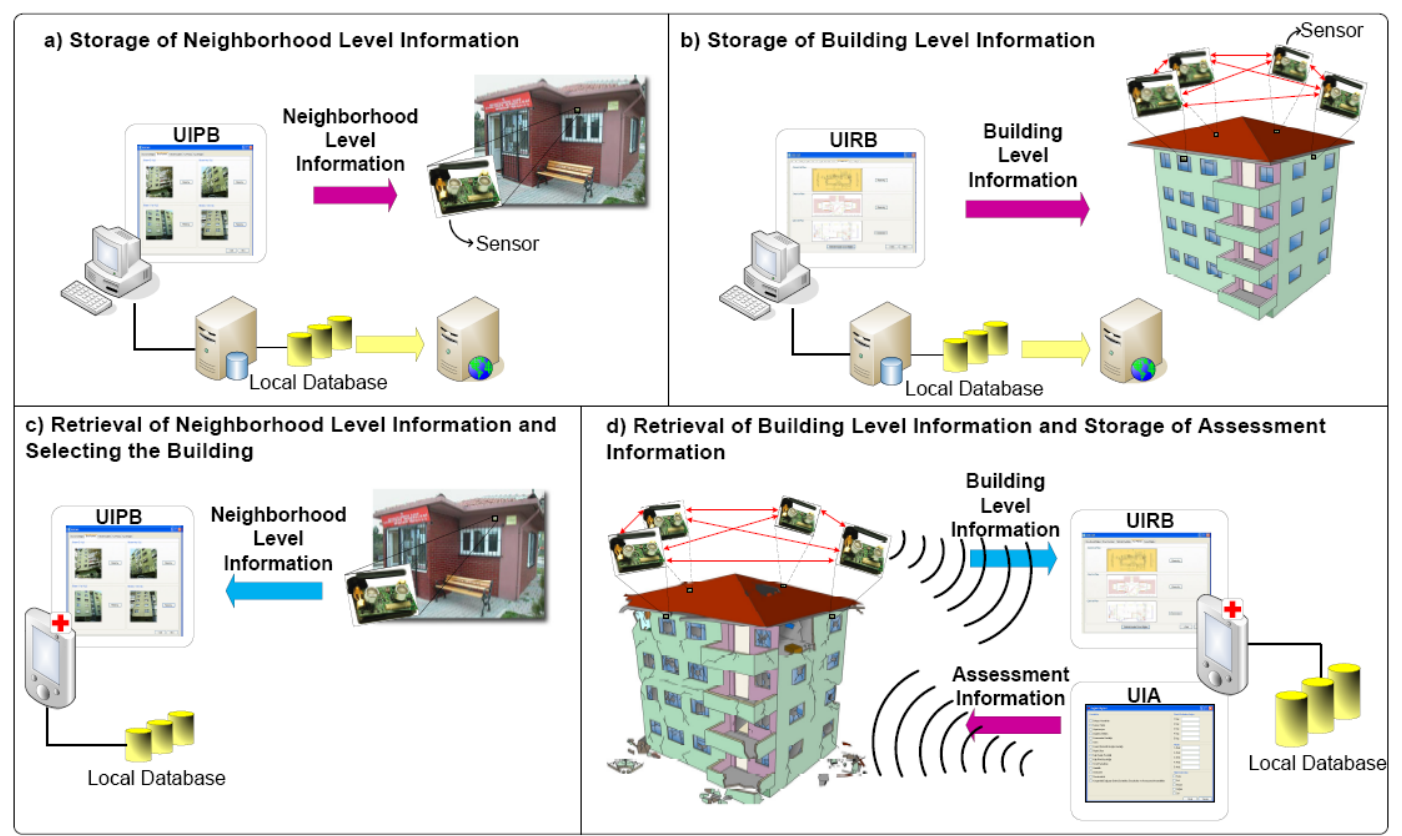

Figure 1: SR-DAP system components and utilization of the system in different phases

\section{Hardware}

SR-DAP was designed and is being implemented by using Sensenode v.1.3 sensors (Figure 2) initially at a laboratory environment. Nodes are compatible with IEEE 
802.15.4 wireless standard and the TinyOS operating system. The low power key characteristic of the sensors is provided by the MSP430 microcontroller having $10 \mathrm{kB}$ RAM and 48kB Flash memory. Sensenode uses the ST M25P80 40MHz serial code flash for external data and code storage. The external flash memory holds $1024 \mathrm{kB}$ of data and is decomposed into 16 segments, each $64 \mathrm{kB}$ in size. Sensenodes have antennas with $80-100 \mathrm{~m}$ range. Onboard chipcon CC240 radio technology provides a $250 \mathrm{kbps}$ data rate at $2.4 \mathrm{GHz}$ frequency (Tsai 2007). Embedded programs for sensors are written in nesC programming language running on TinyOS operating system.

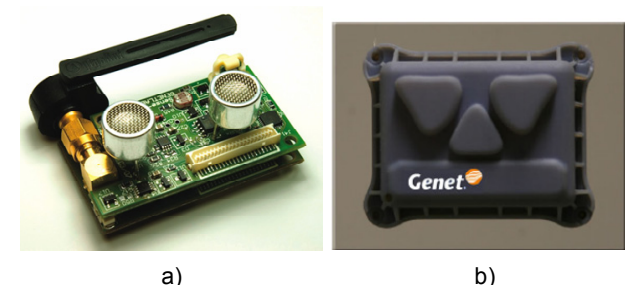

Figure 2: Genetlab Sensenode v.1.3 a) Sensor node b) Casing of the sensor

\section{Data representation}

Some of the information items to be stored in the nodes include media data such as layout plans of the building and pictures of the residents. Since this media data would take up a lot of space on the memory of a sensor node, total storage space needed is minimized to stay within the sensor node's memory capacity limits. For minimizing the storage space; data are encoded into byte streams which are then translated into hexadecimal code for serial transmission. In the encoding procedure, local database entries for specific contents are used to convert the user input into encoded form.

This compression method does not cause any data loss in image files if data is stored in jpeg format. Thus, all image files to be stored are converted into jpeg format. Other compression methods (e.g., Huffman algorithm) are not directly applicable to serial data stream transmission and might cause data loss.

With a proper encoding, all information items including the pictures of the building and the residents can be stored in $661 \mathrm{kB}$ memory space, which is less than the memory space for the sensor node that was used (i.e., $1024 \mathrm{kB}$ ). Total memory requirement was calculated for a building that has twenty floors, four apartments on each floor and 100 residents. Similarly, neighborhood level information is calculated to hold a $455 \mathrm{kB}$ memory space for a neighborhood that has 100 buildings. Much smaller memory space $(1,2 \mathrm{kB})$ is needed for $S \& R$ assessment information which consists of textual data.

\section{Implementation Details}

In the initial tests, data was stored to the flash memory of the sensor node by using the serial link. During serial transmission and storage, data coming from the serial port was written to the flash (external) memory of the node in 256 byte packages. A 256 byte data buffer was defined in the program memory of the sensor and it was used as a temporary storage space for the incoming data from the serial port. Due to single-line serial transmission limitations, piecewise data storage was needed. To implement piecewise storage, writing to external memory and reading from serial port 
was performed separately. More information on data storage can be found at (SarielTalay et al. 2008).

Retrieval of the stored data from the external flash memory of a sensor was performed via a reader sensor connected to a laptop over a serial port. 2048 byte data packages were transmitted to the laptop through wireless communication between two sensors. The sensor storing the data (i.e., the one attached to the building) was the transmitter node and the other sensor connected to the laptop was the receiver node.

After reading the 2048 byte data package from its flash memory, the transmitter sensor sends it to the receiver sensor via a message. The receiver sensor sends the contents of this message to the laptop through the serial port and waits for the next message to come from the transmitter sensor. This process continues until the transmitter sensor completely sends the whole building level information.

The system has three user interfaces to interact with sensors for different operations. User Interface for Residential Buildings (UIRB) is used to enter, update and screen building level information. User Interface for the local Public Buildings (UIPB) is the interface for input/output of neighborhood level information. User Interface for the Assessment Information (UIA) is used by the S\&R team members to store and retrieve assessment information to/from sensors. Currently, building level information is serialized and transmitted to the sensor through the serial line since serial information transfer is faster. Neighborhood and assessment information is transmitted through wireless communication.

These three user interfaces are used in a similar manner for data storage and access, thus one of the user interfaces will be explained as an example in this paper. User Interface for Residential Buildings (UIRB) consists of five forms. Figure 3 shows one of the forms included in the UIRB. In this form, after a hazardous material is selected from a list, the location of that hazardous material is marked on the corresponding layout plan. After all five forms are filled out, the input data is serialized as a single stream and the relevant hexadecimal code is obtained to be transmitted through the serial line to the sensor. The sensor node transfers this incoming hexadecimal code to its flash memory to make it available whenever the node is alive.

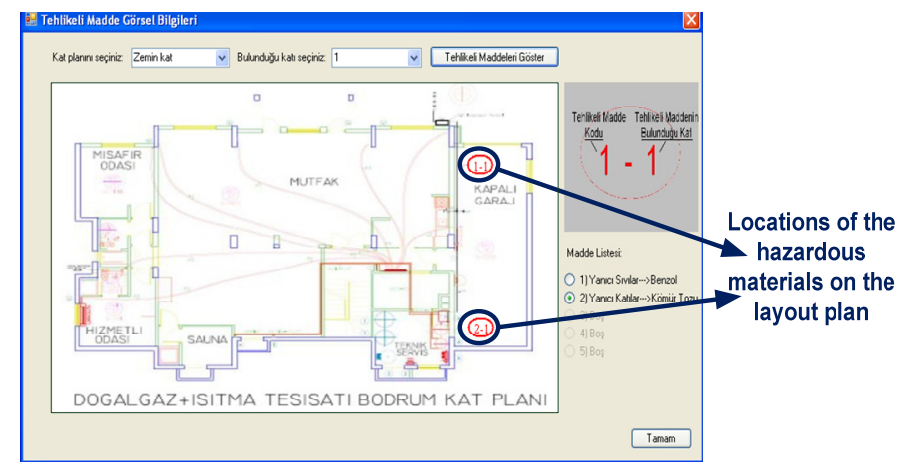

Figure 3: User Interface for entering hazardous material information and its location

To serve for auxiliary purposes as well (e.g., for use in centralized disaster management systems), the given user input is also transmitted to a database server. The user interface can also be used for screening data from the sensor or the database. Local database connectivity is provided for converting user input into byte stream. 


\section{Sensor selection and interaction protocol}

When an S\&R team needs to access building information related to a specific building, multiple sensors belonging to different buildings might be in the range of the receiver. The S\&R team can determine the sensor which stores the target building information by gathering data from all the sensors in range and by going through the pictures or the coordinates of the building which are retrieved from sensors. To perform this filtering, a messaging protocol is developed (Figure 4).

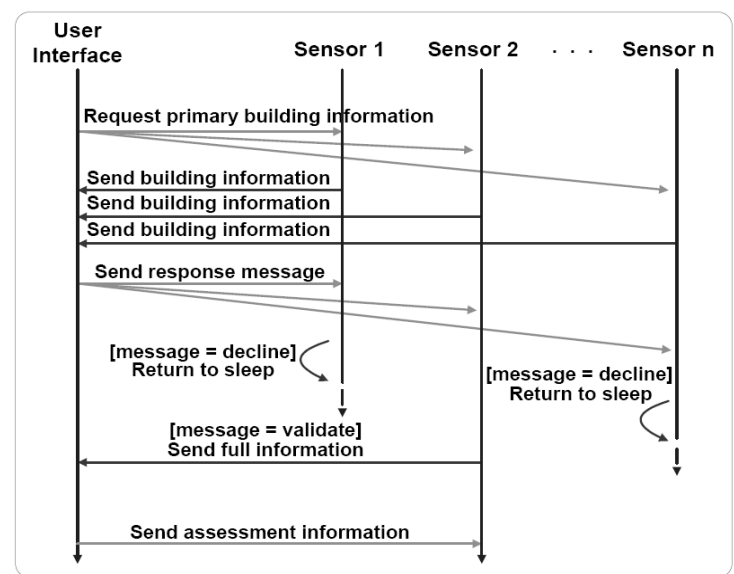

Figure 4: Sequence diagram for the sensor selection and interaction protocol

This protocol consists of several interactions between the reader and the sensors in range. First, a broadcast message is sent to all sensors in the environment querying for the primary information about the buildings (e.g., picture of the building and building name). After receiving this message, sensors in range send the inquired data by extracting them from their external flash memories. The user examines the primary building information sent by multiple sensors and selects the building of interest. This initial phase ends with a validation message sent to the sensor that stores the information related to the building of interest, and decline messages are sent to the rest of the sensors in range. The chosen sensor sends all the data stored in its memory and the rest of the rejected sensors return to sleep mode. After the S\&R operation is performed, the assessment information is stored to the chosen sensor and finally the interaction is completed successfully.

\section{Storage/retrieval of Building Level Information}

An empirical analysis was carried out on a sensor node to measure its reading/writing performance for different sizes of building level information which is directly proportional with number of residents. Figure 5 illustrates the results of this analysis. Figure 5(a) shows the time needed for reading/writing data from/to the sensor with respect to the number of people living in a building and Figure 5(b) shows the data size with respect to the number of people. As expected, writing to the sensor takes more time than reading from the sensor. For a building with 100 people, all the building level information, which holds a $661 \mathrm{kB}$ memory space, is written to the sensor in 34 minutes, and the entire data can be read from the sensor node in 7 minutes. While the reading time is reasonable, the time required for writing to the 
sensor is relatively high. However, note that, writing process is performed before a disaster occurs; thus, there are no time constraints. Data storage after occurrence of a disaster only needed to enter the assessment information of which size is very small.

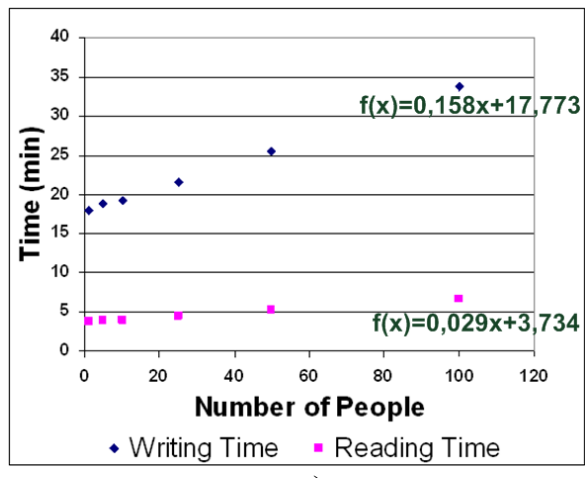

a)

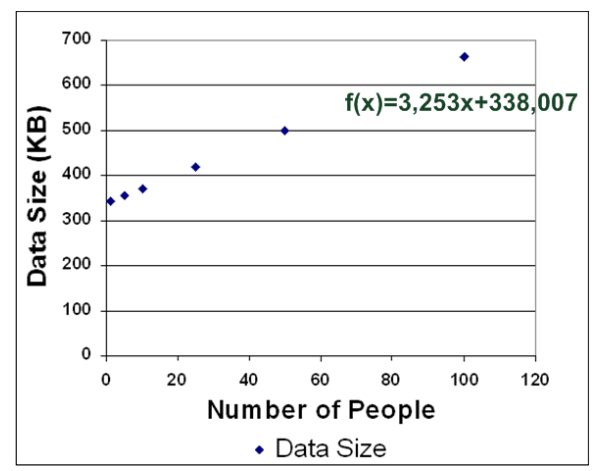

b)

Figure 5: (a) Data read/write time (b) Data size with respect to number of people

\section{Discussion}

Within the proposed system, the local information items are stored as a mixture of media files (e.g., layout plans and resident's pictures) and text information (e.g. personal health information). During data storage and retrieval using wireless sensors, the main challenge was to store large amount of data (i.e., media file) on a limited memory. To minimize the data size, layout plans are saved in jpeg format with low resolution. Storage of a large amount of information is made possible by a proper encoding and compression scheme. To have an efficient data transmission, data was divided into small packages.

For buildings that have many residents, if the size of data to be stored exceeds the amount of the memory space of a sensor, data could be distributed over sensors in a network for each building. Consequently, data retrieval will be performed by collecting all the information distributed over the network. Application of energy consumption schemes by using special storage sensors can be implemented as well (Sheng 2006). Besides, utilizing SD cards on the sensor nodes might be preferred to enlarge the memory capacity of the sensors.

Power consumption of the sensor nodes in the proposed system needs to be determined to figure out average battery life of the sensors. Although battery life of a sensor can be more than 37000 hours (Callaway 2002), the technology provider of the sensor used in this research claims that the battery life is 2000 hours. To estimate power consumption of a sensor node in this application, power requirement in sleep mode and power requirement in data transmission mode in relation to data size need to be identified. If battery life is short, an option would be to connect the sensors to power before a disaster occurs and to only use the battery in case of a power outage. In the proposed system, it is an important task to keep the necessary information upto-date. While information related to a building (e.g., layout plan) mostly remains the same, information related to the residents and contents of a building (e.g., number of people at specific time periods) needs to be updated. To update this information in real time, an efficient tracking mechanism could be implemented by following a similar sensor based approach used for finding lost hikers (Huang et al. 2005). 
However, these techniques are not usually preferred publicly due to privacy concerns. In the SR-DAP system information updates will be performed on a regular basis by a dedicated person in order to provide the updated information to the teams. Sensor nodes do not need to be removed from buildings during this updating process since it is performed through wireless communication.

Although the nodes are encapsulated to be protected against damages caused by a disaster, there is still a risk of data loss caused by an impact or a pressure onto the nodes. Redundant data storage is needed to avoid such a case. For example, assessment information that is entered by an $S \& R$ team to a sensor needs to be transferred to other sensors in the environment to prevent data loss in case of a problem with that sensor. To have data redundancy, sensor network communication architecture needs to be designed to determine how data transfer will be performed.

\section{Conclusions}

This paper gives an overview of the SR-DAP system that is being developed as part of an ongoing research study for storage/retrieval of local information on/from sensor nodes. By using this system, the S\&R operations will be performed more efficiently and effectively since the necessary information will be available on demand.

Within the context of this paper, an overview of information items that need to be stored locally are given including the memory needs for different information groups. The results show that current memory of a single sensor is sufficient for storing required groups of information if proper encoding and compression scheme is used. In addition, design and implementation details of SR-DAP system are presented. The results of the initial laboratory tests, which include the capabilities of data storage and retrieval, sensor communication and data dissemination, are discussed. The findings indicate that time required for reading data from sensors and for writing assessment data to the tags is within reasonable limits, not slowing down S\&R efforts.

Currently tests are being conducted in laboratory environment. The near future work includes field experiments that will be performed to test the designed system in a disaster like environment and implementation of an actual deployment of the system at existing buildings in urban areas. In SR-DAP system, sensors are used as local data storage units and their sensing capabilities are not utilized. In the future, localization of the victims trapped in buildings could also be done dynamically, depending on either carbon dioxide or heat sensing performance of the sensors.

\section{ACKNOWLEDGEMENTS}

This research is funded by a grant from the Scientific and Technological Research Council of Turkey (TUBITAK), Grant No. 107M-144. TUBITAK's support is gratefully acknowledged. Authors also would like to thank Cagri Eroglu and Gurhan Avdan for their contribution.

\section{REFERENCES}

Akyildiz, I. F., Su, W., Sankarasubramaniam, Y., Cayirci, E. (2002). "Wireless Sensor Networks: A Survey", Computer Networks, 38, pp. 393-422.

Asama, H. Hada, Y. Kawabata, K. Noda, I. Takizawa, O. Meguro, J. Ishikawa, K. Hashizume, T. Ohga, T. Hatayama, M. Matsuno, F. Todokoro, 
S. (2006). "Rescue Infrastructure for Global Information Collection", SICE-ICASE, 2006. International Joint Conference, Bexco, Busan, Korea, Oct. 18-21, 2006.

Callaway, E. H. (2002), Wireless Sensor Networks Architectures and Protocols, Auerbach Publications, 1st edition, August 2003, USA

Chen, A.Y., Tsai, M. H., Lantz, Timothy S., Plans, A. P., Mathur, S., Kaushik, N., Lakhera, S., Feniosky, P.M. (2007). "A Collaborative Framework for Supporting Civil Engineering Emergency Response with Mobile Ad-Hoc Networks", ASCE Int. Workshop on Computing in Civil Eng., Pittsburgh, PA, USA, July 25-28, 2007.

Ergen E., Seyis S. (2008). "Utilizing Advanced Storage Technologies For Providing Local Information to Rescue Teams Following an Earthquake", 5th Int. Conference on Innovation in Architecture, Engineering, Antalya, Turkey, June 22-25, 2008

FEMA (2003). "21st Century Complete Guide to FEMA”, U.S. Government, Progressive Management.

Huang J., Amjad S., Mishra S. (2005). "CenWits: A SensorBased Loosely Coupled Search and Rescue System Using Witnesses", The 3rd ACM Conference on Embedded Networked Sensor Systems.

INSARAG (2006). Guidelines and Methodology, Office for the Coordination of Humanitarian Affairs, Field Coordination Support Section (INSARAG Secretariat).

Ito, J. (2006). "Major damage to Asian Internet access from earthquake", Dec. 28, 2006, accessed: Feb. 23, 2009, joi.ito.com/weblog/2006/12/28/major-damage-to.html

Ozerdem, A. and Jacoby T. (2005). "Disaster Management and Civil Society: Earthquake Relief in Japan, Turkey and India", I B Tauris \& Co Ltd.

Pradhan, A, Laefer, D. F., and Rasdorf, W. J. (2007). "Infrastructure Management Information System Framework Requirements for Disasters", Journal Of Computing in Civil Engineering, 21 (2), pp. 90-101.

Sariel-Talay S., Ergen E., Avdan G., and Eroglu C. (2008). "Sensor-Based Data Storage for Search and Rescue", The Fourth Int. Conf. on Intelligent Sensors, Sensor Networks and Information Processing, Sydney, Australia, December 15-18, 2008.

Sheng B., Li Q. and Mao W. (2006) "Data storage placement in sensor networks", 7th ACM Int. Symp.on Mobile Ad-Hoc Networking and Computing, Florence, Italy, May 22-25, 2006.

Tsai, M.-H., Liu, L. Y., Feniosky, P, M. (2007). "A Building Black Box for Urban Disaster Response and Relief," ASCE International Workshop on Computing in Civil Engineering, Pittsburgh, PA, USA, July, 25-28, 2007.

Worldbank (1999). "Turkey - Marmara earthquake assessment", Turkey Country Office, The World Bank, http://siteresources.worldbank.org, September 14, 1999. 